\title{
Natural Glycoside Salidroside Ameliorates Orthopedic Surgery-Induced Cognitive Dysfunction Through Activating Adenosine 5'-Monophosphate- Activated Protein Kinase Signaling in Mice
}

\section{Cai-Long Pan}

Nanjing Medical University

Guo-Liang Dai

Affiliated Hospital of Nanjing University of Chinese Medicine: Jiangsu Province Academy of Traditional

Chinese Medicine

Hui-Wen Zhang

Nanjing Medical University

Chen-Yang Zhang

Nanjing Medical University

Qing-Hai Meng

Nanjing University of Chinese Medicine

Lu Xu

Nanjing Medical University

Ning $X u$

Nanjing Medical University

Xue Liu

Nanjing Medical University

Zhiren Zhang

Army Medical University

Yong Zhang

Nanjing Medical University

Qi-Lian Tan

Nanjing Medical University

Xiao-Liang Wang

Nanjing Medical University

Zhi-Yuan Zhang ( $\nabla$ zzy@njmu.edu.cn )

Nanjing Medical University https://orcid.org/0000-0001-9067-0670 
Keywords: salidroside, microglia, perioperative neurocognitive disorders, AMPK, PPARY

Posted Date: August 3rd, 2021

DOI: https://doi.org/10.21203/rs.3.rs-745010/v1

License: (c) (1) This work is licensed under a Creative Commons Attribution 4.0 International License. Read Full License 


\section{Abstract}

Background: Perioperative neurocognitive disorders (PND) are the most common postoperative complications with few therapeutic options. Salidroside, a plant-derived compound, has gained increased attention as treatment for various neurological diseases and particularly modifier of microglia-mediated neuroinflammation. However, the effect of salidroside on orthopedic surgery-induced cognitive dysfunction and the underlying mechanisms are largely unknown.

Methods: The Morris water maze test was used to investigate potential effects of salidroside in the animal model of tibia fracturing with intramedullary fixation. Therapeutic mechanism and related signaling pathways of salidroside in PND were further investigated with animal tissues and microglial cultures in vitro by molecular biology tests.

Results: Here we found that salidroside greatly attenuated cognitive impairment in mice after orthopedic surgery. Neuroinflammation in mouse hippocampus were also attenuated by salidroside. Meanwhile, salidroside treatment induced a switch in microglia polarization to the anti-inflammatory phenotype. In vitro, salidroside suppressed the expression of pro-inflammatory cytokines and induced a switch in microglial phenotype to the anti-inflammatory phenotype. Mechanically, molecular docking studies revealed potential AMPK activation activity of salidroside. And salidroside did up-regulated the AMPK pathway proteins. Moreover, AMPK antagonist abolished the effects of salidroside in vivo and in vitro.

Conclusions: Taken together, our results demonstrated that salidroside effectively suppressed PND by suppressing microglia-mediated neuroinflammation through activating AMPK pathway, and it might be a novel therapeutic approach for PND.

\section{Introduction}

Perioperative neurocognitive disorders (PND) are the most common central nervous system complication that occurs during the perioperative period, manifested as postoperative changes in social and cognitive ability, even longer-lasting cognitive decline, especially after cardiac and orthopedic surgery [1]. Compared with young patients $(36.6 \%)$, elderly patients $(41.4 \%)$ had a higher incidence of postoperative cognitive dysfunction at hospital discharge and higher risk of long-term cognitive impairment [2]. As global aging situation intensifies, more elderly patients will inevitably be exposed to surgical treatment. PND imposes a heavy burden on individuals and society, including prolonged hospital stay, increased mortality, decreased quality of life of patients after surgery, and consumption of medical resources $[3,4]$. Neuroinflammation is considered to be one of the important mechanisms leading to PND, in which microglia play a key role [5]. Preclinical and clinical studies have proved that microglia mediate postoperative neuroinflammation and cognitive dysfunction in patients and rodents [6-9]. Perioperative microglial depletion by the colony-stimulating factor 1 receptor inhibition or minocycline significantly protected mice from PND [6, 7]. However, there are currently few approved drugs for clinical treatment of PND. Therefore, effective treatment options were urgently needed. 
Rhodiola rosea is one of the tonic herbs in traditional Chinese medicine and have long been used for antidepressant, anti-fatigue and anti-stress in Asia and Europe [10,11]. Studies report that Rhodiola rosea has beneficial effects for the treatment of cognitive dysfunction through the suppression of oxidative stress and neuroinflammation in brain [12]. Recent study has demonstrated that the extract of Rhodiola rosea inhibited the neuroinflammation in response to stress through mitogen-activated protein kinases (MAPK) and nuclear factor kappa B (NF-KB) signaling pathway [13]. Salidroside is the main active ingredient of the Rhodiola rosea, with multiple functions, including cardiopulmonary protection, antifatigue, pain relief, anti-inflammation, and neuroprotection $[12,14]$. For example, salidroside reduced the expression of inflammatory cytokines induced by lipopolysaccharide (LPS), ultraviolet or interleukin-1 $\beta$ (IL-1 $\beta$ ) in different cells [15-17]. In an animal model of spinal cord injury, salidroside promoted motor function recovery by reducing the inflammatory response, and its mechanism involved several signaling pathways such as adenosine $5^{\prime}$-monophosphate-activated protein kinase (AMPK), NF-қB, and extracellular-signal regulated kinase (ERK)/MAPKs $[18,19]$. Recently, accumulating researches have shown that salidroside showed a neuroprotective effect against $A \beta$-induced neurotoxicity and improved behavioral performance in drosophila and mouse Alzheimer's disease (AD) models [20,21]. Salidroside reduced cerebral infarction and improve neurological function in animal models of cerebral ischemia, and prevent cognitive dysfunction caused by cerebral hypoxia or hypoperfusion, which was attributed to modulating the phosphoinositide 3-kinase (PI3K), NF-KB, MAPK, hypoxia-inducible factor a (HIFa) or matrix metalloproteinases (MMPs) signaling pathway [22]. Salidroside improved LPS-induced learning and memory impairments and neuroinflammation by SIRT1-dependent nuclear factor erythroid 2-related factor 2 (Nrf-2) / heme oxygenase-1 (HO-1) /NF-KB pathway [23]. However, few studies have reported the effect of salidroside on orthopedic surgery-induced cognitive dysfunction.

Evidence suggests that the risk of fractures increases sharply in elder [24]. The fracture repair or hip arthroplasty is one of the most common surgical operation for the elderly, and orthopedic surgery is one of the main causes of PND [25]. Therefore, an orthopedic surgery mouse model of open tibial fracture with intramedullary fixation was used to investigate potential therapeutic effect of salidroside on PND here. In this study, we aim to systemically examine the inhibitory effects of salidroside on cognitive dysfunction and neuroinflammation in mice received tibia fractures with intramedullary fixation, which meeting the characters of clinical surgery such as fracture repair, and further explore the underlying mechanisms.

\section{Materials And Methods}

\section{Reagents}

Salidroside (purity: 99\%, CAS: 10338-51-9) was purchased from Mansite Bio-technology (Chengdu, China). Metformin, rosiglitazone, GW9662, and Compound C were purchased from Sigma-Aldrich (St. Louis, MO, United States). Antibodies for IBA-1 and PPARy were purchased from Abcam (Cambridge, United Kingdom). Antibodies for AMPK, p-AMPK (Thr172), NF-KB p65, CD86 were purchased from Cell Signaling Technology (MA, United States). Antibody for CD163 and GAPDH, and all second antibodies 
were purchased from Proteintech (Wuhan, China). HE staining kit, DAB kit, and hypersensitive ECL chemiluminescence kit were purchased from Solarbio (Beijing, China). Other reagents were purchased from Sinopharm Reagent Group Co. Ltd (Shanghai, China).

\section{Molecular docking}

Schrödinger (2018) was used to conduct molecular docking between AMPK and salidroside to explore the binding mode. Firstly, crystal structure of AMPK from the (PDB:4CFE) was get from https://www.rcsb.org/. The structure of AMPK was imported into the Protein Preparation Wizard module to optimize protein structure, for removing all crystal water molecules, correcting side chains with missing atoms, adding hydrogen atoms. Then using the OPLS3 force field to distribute protonated states and partial charges. The crystal structure energy was minimized until the root mean square deviation (RMSD) of the non-hydrogen atoms reached $0.3 \AA$. After that, the LigPrep module was used to optimize 11 small ligand molecules to generate ionization and tautomerism states between $\mathrm{pH} 6.8$ and 7.2. The OPLS3 force field was again used to minimize the energy of small molecules, the maximum number of stereoisomers was set to 32. Finally, the Receptor Grid Generation module was used to generate lattice files for docking. Glide module was used for molecular docking, and Extra Precision (XP) was used for analyzing. The docking score was recorded to assess the docking effect between salidroside and AMPK.

\section{Animals and orthopedic surgery model}

Adult male C57BL/ 6 mice ( 15 months of age) were provided by the Experimental Animal Center at Nanjing Medical University, Nanjing, China. All experimental protocols and procedures were approved and licensed by Nanjing Medical University Animal Care and Use Committee in accordance with the National Institutes of Health guide for the care and use of Laboratory animals.

Orthopedic surgery was imitated by the model of tibial fracture with intramedullary fixation [26]. The mice were anesthetized with sodium pentobarbital $(35 \mathrm{mg} / \mathrm{kg})$. The left hind limb of the mouse was shaved and disinfected. After the tibia was exposed, a $0.3 \mathrm{~mm}$ pin was inserted into the tibial medullary cavity. Then the fracture occurred, and the skin was sutured with 4.0 nylon. Lidocaine cream was used locally for analgesia. The mice in sham group received all perioperative operations, except the tibial fracture and intramedullary fixation. The mice in surgery + salidroside group were intraperitoneally injected with different concentrations of salidroside $(20,40,80 \mathrm{mg} / \mathrm{kg}$, once a day) for 5 days before surgery.

\section{Morris water maze (MWM) test}

The MWM test was performed as described previously [27]. Briefly, a transparent cylindrical escape platform with a diameter of $10 \mathrm{~cm}$ was placed in one quadrant of $1.25 \mathrm{~m}$-diameter circular water pool filled with water, and the platform was $1 \mathrm{~cm}$ below the water surface. The latency was recorded, which is 
the time it takes for the mouse to enter the water and climb onto the platform. If the mouse did not find the platform within $60 \mathrm{~s}$, it would be guided to the platform, stay for $20 \mathrm{~s}$, and then be taken out. The experiment was carried out at the same time every day for 5 consecutive days. On the 6th day, the platform was removed to start the probe test, and the time for the mouse to enter the target quadrant and the crossing number of the position of the platform within $60 \mathrm{~s}$ was recorded.

\section{Section and staining of brain tissue}

After the mice were sacrificed, their brains were taken out and fixed in $4 \%$ paraformaldehyde for $24 \mathrm{~h}$. After the fixed mouse brain tissue was dehydrated, it was soaked in xylene and placed in paraffin melted at $65^{\circ} \mathrm{C}$ for $4 \mathrm{~h}$. Then, the mouse brain tissue was prepared into a wax block using a paraffin embedding machine. Mouse brain tissue was sliced into $5 \mu \mathrm{M}$ slices using a paraffin rotary microtome. After the paraffin sections were baked, they were deparaffinized and then soaked in xylene for $10 \mathrm{~min}$. After washing and soaking with absolute ethanol, the slices were soaked in ethanol solution of decreasing concentration until placed in water.

As for hematoxylin-eosin (HE) staining, mouse brain tissue slices were covered with hematoxylin staining solution, washed after $10 \mathrm{~min}$, differentiated in $1 \%$ hydrochloric acid-ethanol solution, and fully turned blue in dilute ammonia. The slices were washed again and covered with eosin staining solution for 1 min. The excess staining solution on the brain tissue was washed away. After the slices were rapidly dehydrated, they were soaked in xylene for $5 \mathrm{~min}$, and then mounted with a neutral resin slicing solution. The slices were placed under an optical microscope for observation and photographs were taken.

As for immunohistochemical and immunofluorescence staining, mouse brain tissue slices were soaked in $3 \%$ hydrogen peroxide solution for $20 \mathrm{~min}$. After washing with PBS, the slices were immersed in a $0.01 \mathrm{M}$ sodium citrate solution with a pH of 6.0 after heating to $95^{\circ} \mathrm{C}$ for $20 \mathrm{~min}$. After natural cooling, the slices were covered with $5 \%$ BSA. After 20 min, the BSA solution was removed.

As for immunohistochemical staining, the slices were incubated with the primary antibody, IBA1 (1:100), CD86 (1:100), CD163 (1:100), and incubated overnight at $4^{\circ} \mathrm{C}$ in the refrigerator. The next day, after washing with PBS, the slices were incubated with diluted HRP-conjugated secondary antibody (1:100) for $2 \mathrm{~h}$. After washing again, the slices were developed with DAB color reagent, and washed with water immediately after full color development. The brain tissue was covered with hematoxylin staining solution, washed after $10 \mathrm{~min}$, and then fully returned to blue in dilute ammonia after differentiation with $1 \%$ hydrochloric acid-ethanol solution. The slices were washed again, and after rapid dehydration, the slices were soaked in xylene for $5 \mathrm{~min}$, and then mounted with a neutral resin slicing solution. The slices were placed under an optical microscope for observation and photographs were taken.

As for immunofluorescence staining, the slices were incubated with the first antibody, goat-IBA1 (1:100) and rabbit-AMPK (1:100), or goat-IBA1 (1:100) rabbit-PPARY (1:100), overnight at $4{ }^{\circ} \mathrm{C}$. On the second day, after the slices were washed with PBS, they were incubated with TRITC-labeled rabbit-anti-goat IgG and 
FITC-labeled goat-anti-rabbit-IgG for $2 \mathrm{~h}$. After washing, the slices were incubated with DAPI staining solution for $5 \mathrm{~min}$. After washing, the slices were mounted with glycerin gelatin mounting solution. The expression of IBA1 and AMPK or PPARY was observed with a fluorescence microscope, photographed, and analyzed.

\section{Cell culture and viability measurements}

BV-2 cells were cultured in DMEM containing 10\% fetal bovine serum, $100 \mathrm{U} / \mathrm{mL}$ penicillin, and 100 $\mathrm{mg} / \mathrm{mL}$ streptomycin in a $37^{\circ} \mathrm{C}$ incubator containing $5 \%$ CO2. The cells were treated with LPS $(1 \mu \mathrm{g} / \mathrm{ml})$ with or without salidroside $(25,50$, and $100 \mu \mathrm{M})$ for $12 \mathrm{~h}$. Cell extracts and supernatants were collected and analyzed.

As for cell viability, after the cells were treated with or without salidroside $(25,50$, and $100 \mu \mathrm{M})$ for $12 \mathrm{~h}$, LPS $(1 \mu \mathrm{g} / \mathrm{ml})$ was used to induce the BV-2 cells for $24 \mathrm{~h}$, after that the CCK-8 reagent (10 ul) was added to each well. After $4 \mathrm{~h}$, the absorbance value of each well was measured at $450 \mathrm{~nm}$ using a microplate reader. According to the formula, cell viability $=(\mathrm{OD}$ value of detection well $/$ mean $\mathrm{OD}$ of control wells) $\star 100$.

\section{Quantitative real-time reverse transcription-polymerase chain reaction ( $R T-q P C R)$}

For the brain tissues of the sacrificed mice and the cells collected after treating with or without salidroside for $12 \mathrm{~h}$ followed by LPS for $24 \mathrm{~h}$, Trizol reagent was used to obtain the total RNA. After the addition of isopropanol, the supernatant was collected, and the RNA was precipitated with ethanol, and then the mRNA of mouse brain tissue and BV-2 cells was reverse transcribed into cDNA using a reverse transcription kit. The primers of IL-1 $\beta$, IL-4, IL-6, IL-10, IL-12, TNF- $a$, IFN- $y$, CCL5, CXCL1, CXCL10, CD16, TGF- $\beta$, Arg1, YM1, and GAPDH were provided in table 1 . These primers were synthesized and provided by Biotech Bioengineering (Shanghai) Co., Ltd. The method was carried out with reference to the instructions of One Step TB Green ${ }^{\circledR}$ PrimeScript ${ }^{\text {TM }}$ RT-PCR Kit II (Cat. No. RR086A/B, Baori Biotechnology (Beijing) Co., Ltd., Beijing, China), and the gene expression was analyzed using the ABI7500 system.

\section{Enzyme-linked immune sorbent assay (ELISA)}

After the cells were treated with or without salidroside for $12 \mathrm{~h}$, LPS $(1 \mu \mathrm{g} / \mathrm{ml})$ was used to induce the BV2 cells for $24 \mathrm{~h}$, then the supernatant of the cells was collected. The levels of TNF-a, IL-1 $\beta$, IL- 6 , and IL-10 in these supernatants were detected using mouse TNF-a, IL-1 $\beta$, IL-6, and IL-10 ELISA detection kits, referring to the instructions. TNF- $a$, IL-1 $\beta$, IL- 6 , and IL-10 ELISA test kits were purchased from Solarbio (Beijing, China). 


\section{Western blot}

For the brain tissues of the sacrificed mice and the cells collected after treating with or without salidroside for $12 \mathrm{~h}$ followed by LPS for $24 \mathrm{~h}$, after lysis with Protein Lysis Buffer, the supernatant was collected after centrifugation at $12000 \mathrm{~g} / \mathrm{min}$ for $10 \mathrm{~min}$. After the sample was prepared, it was added to the SDS-PAGE gel and the proteins were separated by electrophoresis. The protein was transferred to the PVDF membrane, and after being fully blocked, PVDF membrane was incubated with the diluted antibodies against AMPK (1:2000), p-AMPK (1:1000), PPARY (1:2000), and GAPDH (1:10000) at $4^{\circ} \mathrm{C}$ overnight. After the PVDF membrane was washed, it was incubated with the HRC-conjugated Goat AntiRabbit IgG for $2 \mathrm{~h}$. Then a gel imaging system and a chemiluminescence kit were used to obtain the western blot photos.

\section{Nuclear translocation of NF-kB p65}

BV-2 cells were seeded into a 6-well plate with glass slides for cell climbing. After the cells were treated with or without salidroside for $12 \mathrm{~h}$ followed by LPS for $24 \mathrm{~h}$, all cells were fixed with paraformaldehyde. After washing, the cells were covered with diluted anti-NF-KB p65 (1:100) antibody and incubated overnight. After washing the cells with PBS, the cells were covered with diluted Fluorescein (FITC)conjugated Goat Anti-Rabbit IgG (1:100) and incubated for $2 \mathrm{~h}$. After multiple washing, the cells were stained with DAPI and incubated for $5 \mathrm{~min}$. After washing the BV- 2 cells for at least 3 times, the cell slide was taken out, and the cell was covered on the glass slide using glycerin gelatin mounting solution. A fluorescence microscope was used to take pictures of cells.

\section{Statistics}

Image pro plus6.0 was used to analyze immunofluorescence and immunohistochemistry pictures and export measurement data. Quantity One was used to analyze the blot data and perform relative quantitative analysis. After all the data were derived from the measurement data, the SPSS 23.0 statistics were used to perform a one-way analysis of variance on the measurement data first logarithm, and the measurement data are expressed as the mean \pm standard deviation. $\mathrm{P}<0.05$ indicated that the difference was statistically significant. Bonferroni's posttest conducted the post hoc test. GraphPad Prism 7.0 (San Diego, CA, USA) was used to graph the measurement data.

\section{Results}

\section{Salidroside ameliorates orthopedic surgery-induced memory deficits}


First, the effect of salidroside on cognitive impairment caused by orthopedic surgery was tested. The Morris Water Maze (MWM) test is used to evaluate the locomotor ability and cognitive behavior of mice. The results showed that the swim speed in 7 to 12 days after orthopedic surgery between the groups has no significant different (Figure 1A), indicating that there is no significant difference in the locomotor activities of each group mice. Mice in the surgery group showed a significantly increased escape latency compared to the sham group (Figure 1B). In the probe test, the platform crossing time and the time spent in the target quadrant of the mice in the surgery group were significantly reduced, compared with mice in the sham group (Figure 1C-E). The salidroside treatment significantly improved the above behavioral performance in a dose-dependent manner $(20,40$ and $80 \mathrm{mg} / \mathrm{kg})$, including the latency, platform crossing time and the time spent in the target quadrant (Figure 1B-E). We then assessed the histological changes of the mice using HE staining. As shown in Figure 1F, no abnormalities in brain cells were observed in the sham group with or without $80 \mathrm{mg} / \mathrm{kg}$ salidroside treated. In contrast, nuclear pyknosis was observed in the surgery group. Moreover, orthopedic surgery mice treated with salidroside showed significantly fewer abnormal cells compared to the surgery group.

\section{Salidroside inhibits orthopedic surgery- induced neuroinflammation in mice}

Then, we tested the effect of salidroside on orthopedic surgery-induced neuroinflammation by detecting the expression of inflammatory factors and the activation state of microglia in the hippocampus of mice. The mRNA levels of pro-inflammatory cytokines TNF- $a, I L-1 \beta, I L-6$ and anti-inflammatory cytokine IL-10 in the hippocampus were detected by RT-qPCR. The results showed that the levels of TNF-a, IL-1 $\beta$, and IL- 6 were significantly increased and the levels of IL-10 was significantly decreased in the hippocampus of mice after surgery, while the administration of salidroside $(80 \mathrm{mg} / \mathrm{kg})$ in orthopedic surgery mice observably prevented the above changing of mRNA expression in inflammatory factors (Figure 2A). Further, we used immunohistochemical staining to detect the activation and status of microglia, and the results showed that orthopedic surgery induced prominent IBA1 expression in the hippocampus of mice, while salidroside significantly reduced the IBA1 expression in surgery mice (Figure 2B-C). Salidroside significantly reduced the expression of CD86 in the hippocampus, indicating that salidroside treatment reduced the number of $\mathrm{M} 1$ microglia in orthopedic surgery mice (Figure 2D-E). We also performed immunohistochemical staining on the M2 microglia marker CD163, which was increased after salidroside treatment (Figure 2D and 2F). These data indicate that salidroside inhibited neuroinflammation and induced M2 microglia polarization.

\section{AMPK and PPARy participate in the neuroprotective effect of salidroside in PND mice}

Next, we examine the possible mechanism of salidroside on the neuroprotection of PND. It has been reported that AMPK and peroxisome proliferator-activated receptor y (PPARY) were involved in microglia- 
mediated neuroinflammation [28, 29]. Recent studies have indicated that AMPK signaling is related to the beneficial effects of salidroside in diabetes [30]. Therefore, we hypothesized that salidroside might suppress orthopedic surgery-induced neuroinflammation and cognitive impairment by activating AMPK/PPARy signaling pathway. Molecular docking confirmed that salidroside formed hydrogen bonds with amino acids H323 and C285 of AMPK, which may partially extract the activation capacity on AMPK, and thus initiating APMK phosphorylation. The docking score between salidroside and AMPK was -8.074 (Fig.3A). Further, AMPK phosphorylation in mouse hippocampus was significantly increased after salidroside treatment (Figure 3B-C). The immunofluorescence results also showed that salidroside remarkably activated AMPK, which is co-localized with IBA $1^{+}$microglia (Figure $3 \mathrm{~F}-\mathrm{G}$ ). In addition, the results of western blot showed that salidroside promoted the expression of PPARY (Figure 3D-E), which is consistent with the results of immunofluorescence staining, and the activation of PPARY was co-localized with IBA $1^{+}$microglia (Figure $\left.3 \mathrm{H}-\mathrm{I}\right)$. These data indicate that salidroside could activate the AMPK and PPARY in microglia in mouse hippocampus.

\section{Salidroside inhibits LPS-induced inflammatory response in BV-2 cells}

Further, we tested the direct effect of salidroside on the cultured microglia cell line BV-2 cells in vitro. The results showed that salidroside did not cause cytotoxicity in normal BV-2 cells at a concentration of 1 $500 \mu \mathrm{M}$ (Figure 4A). And $1 \mu \mathrm{g} / \mathrm{ml}$ LPS incubation induced prominent inflammatory response in BV-2 cells, the secretion of TNF- $a, \mathrm{IL}-6$, and IL-1 $\beta$ increased, and the secretion of IL-10 decreased significantly (Figure $4 C-F)$. The cell viability of BV-2 cells in salidroside $(25,50$, and $100 \mu \mathrm{M})$ and LPS treatment condition had no significant change (Figure 4B), however, salidroside dose-dependently $(25,50$, and $100 \mu \mathrm{M})$ reduced the secretion of TNF-a, IL-1 $\beta$ and IL- 6 secreted by BV- 2 cells into the culture medium induced by LPS, and increased the secretion of IL-10 (Figure 4C-F). The results of immunofluorescence showed that salidroside $(100 \mu \mathrm{M})$ prevented LPS-induced nuclear translocation of NF-KB p65 (Figure 4G-H). These results showed the direct anti-inflammatory effect of salidroside in microglia. To reveal the characteristics of microglia activation after salidroside treatment, M1 microglia marker CD86, and M2 microglia marker CD163 were examined. As show in Figure 4I-J, $100 \mu \mathrm{M}$ salidroside decreased the CD86 expression and increased CD163 expression in LPS-induced BV-2 cells. These results showed that salidroside inhibited LPS-induced inflammatory response of microglia and promoted its polarization to the M2 phenotype.

\section{The AMPK/PPARy pathway is involved in anti-inflammatory effect of Salidroside in BV-2 cells}

LPS $(1 \mu \mathrm{g} / \mathrm{ml})$ incubation reduced the p-AMPK and PPARy protein expression in BV-2 cells, while salidroside $(100 \mu \mathrm{M})$ increased the expression of p-AMPK and PPARY in LPS induced BV-2 cells (Figure 5A-B). Two clinically commonly used drugs that induce AMPK and PPARY activation respectively, metformin and rosiglitazone, were used as positive controls. Like salidroside, they both increased the 
protein expression levels of p-AMPK and PPARy in LPS induced BV-2 cells (Figure 5C-D). In LPS induced BV-2 cells, the secretion of TNF- $a, \mathrm{IL}-6, \mathrm{IL}-1 \beta$, and IL-10 were also affected by salidroside, metformin and rosiglitazone, they all reduced TNF-a, IL-6, and IL-1 $\beta$ level, and increased IL-10 level in cell culture supernatant (Figure 5E-H). These results indicated that AMPK and PPARY activation reduced microglia inflammation, and AMPK and PPARY were involved in the effect of salidroside on microglia.

Next, we tested the effect of salidroside on the inflammation and differentiation of microglia under the condition of AMPK or PPARy inhibition. As show in Figure 6A-B, salidroside significantly increased AMPK phosphorylation and PPARY expression in BV-2 cells, the promoting effect of salidroside on PPARY activity was blocked by AMPK inhibitor compound $C$, but promoting effect of salidroside on AMPK phosphorylation had no significant difference in LPS induced BV-2 cells with or without PPARY antagonist GW9662 treatment (Figure 6A-B). These results indicate that AMPK and PPARY activation were involved in the effect of salidroside on microglia, and AMPK was upstream of PPARY in the effect of salidroside on microglia. The anti-inflammatory effect of salidroside, reducing TNF- $\alpha$, IL- 6 , and IL-1 $\beta$ level, and increasing IL-10 level in cell culture supernatant in LPS induced BV-2 cells, were blocked by PPARY antagonist GW9662 and AMPK inhibitor compound C (Figure 6C-F), which indicating the inhibition of LPS-induced inflammatory response in BV-2 cells by salidroside needs the activation of AMPK and PPARy. In LPS induced BV-2 cells, salidroside, metformin or rosiglitazone treatment decreased CD86 expression and increased CD163 expression (Figure 6G-H). The regulation of microglia polarization of salidroside, decreasing CD86 expression and increasing CD163 expression, were blocked by PPARY antagonist GW9662 and AMPK inhibitor compound C (Figure $6 \mathrm{G}-\mathrm{H}$ ), which indicating the regulation of microglia polarization by salidroside needs the activation of AMPK and PPARY. To further reveal the characteristics of microglia activation after salidroside treatment, M1 microglia marker genes (IL-1 $\beta$, IL12, TNF-a, IFN- - , CCL5, CXCL1, CXCL10, and CD16), and anti-inflammatory cytokines - M2 microglia marker genes (IL-10, IL-4, TGF- $\beta$, Arg1, and YM1) were examined in BV-2 cells. As show in Figure 6I, salidroside, rosiglitazone, or metformin decreased the expression of LPS-induced M1 phenotypic marker genes and increased the expression of M2 marker genes. The PPARY antagonist GW9662 and AMPK inhibitor compound $\mathrm{C}$ prevented salidroside-mediated decrease in $\mathrm{M} 1$ marker gene expression and increase in M2 marker gene in LPS-induced BV-2 cells (Figure 6l). These results indicated that AMPK and PPARY activation reduced microglia inflammation, and salidroside inhibits LPS-induced inflammatory response of microglia and promotes its polarization to the M2 phenotype via activating AMPK/PPARY pathway.

\section{The effect of salidroside in improving cognitive impairment in mice depends on AMPK}

Finally, we verified in vivo whether the neuroprotective effect of salidroside depends on AMPK. The MWM test is used to evaluate the locomotor ability and cognitive behavior of mice. The results showed that the swim speed in 7 to 12 days after orthopedic surgery between the groups has no significant different (Figure 7A). Salidroside significantly inhibited the escape latency of mice in surgery mice and increased 
the platform crossing numbers and time spent in target quadrants, which could be canceled by AMPK inhibitor compound C (Figure 7B-E). The immunohistochemical staining was used to detect the PPARY expression and activation of microglia. The results showed that AMPK inhibitor compound $\mathrm{C}$ canceled the suppress effect of salidroside on IBA1 expression, and canceled the promotion effect of salidroside on PPARy expression in the hippocampus of surgery mice (Figure 7F-H), which is consistent with in vitro results. In addition, salidroside decreased the expression of M1 phenotypic marker genes (IL-1 1 , IL-12, TNF-a, IFN- $y$, CCL5, CXCL1, CXCL10, and CD16) and increased the expression of M2 marker genes (IL-10, IL-4, TGF- $\beta$, Arg1, and YM1) in orthopedic surgery-induced cognitive impairment mice, which was abolished by AMPK inhibitor compound C (Figure 7I). These results indicate that salidroside improved the cognitive impairment of orthopedic surgery mice by AMPK/PPARY signaling pathway.

\section{Discussion}

Herein, we demonstrated that salidroside prevents PND by inhibiting neuroinflammation in a mouse model of tibia fractures with intramedullary fixation. Salidroside shows a further beneficial effect by promoting the polarization of microglia to the anti-inflammatory phenotype. In addition, we also analyze the potential signaling mechanism of salidroside to exert its beneficial effects: promote the activation of AMPK/PPARY. Salidroside activated the AMPK and PPARY in the hippocampus of mice and BV-2 cells. More importantly, the effects of salidroside that mitigated the cognitive deficit in mice induced by orthopedic surgery and promoted PPARy activation and microglia polarization were abolished by AMPK antagonist. Taken together, salidroside prevented surgery-induced neuroinflammation and cognitive deficits via the AMPK/PPARY signaling pathway and might be a novel therapeutic approach for PND.

Recently, an increasing number of studies have been conducted on the neuroprotective effects and cognition enhancement of salidroside [14]. It was demonstrated that salidroside ameliorated neurodegeneration and memory impairment in hypoxic rats and $A \beta 1-40$-injected $A D$ rat model $[31,32]$. Salidroside has shown protective effects in behavior tests and hippocampal long-term potentiation tests in rats with cognitive deficits caused by chronic cerebral hypoperfusion. Salidroside also ameliorated arthritis-induced cognition dysfunction by decreasing pro-inflammatory cytokines in hippocampus and serum [33]. However, the underlying molecular mechanism remains unclear. In the present study, we found that salidroside prevented the cognitive function of orthopedic surgery mice (Fig. 1), which were in line with the findings of another study which demonstrating that salidroside was effective against in isoflurane-induced learning and memory impairment [34]. And it was suggested that the underlying mechanism of the effect of salidroside was related to the inhibition of excessive inflammatory response, decrease of oxidative stress, and regulation of the cholinergic system [34]. Our research proved that the neuroprotective effect of salidroside depends on the activation of AMPK/PPARY signaling pathway.

Increasing evidence has shown that the AMPK signaling pathway plays a crucial role in the pathophysiology of neurodegenerative diseases $[35,36]$. AMPK is a Ser/Thr kinase which has a crucial role in the maintenance of energy metabolism and anti-inflammation. AMPK activators have shown beneficial effects in AD models, such as improved cognitive deficits, reduced $A \beta$ production in a 
transgenic AD mouse model [37]. Moreover, AMPK activation in brain increased microglial M2 gene expression in an LPS-induced neuroinflammation mouse model [38]. Consistent with these studies, our results show that the AMPK agonist metformin significantly improved cognitive deficits after surgery and reduced the LPS-induced inflammatory response in BV-2 cells, which mimic the effect of salidroside. Moreover, PPARy has been found to play an essential role in cognitive performance [39]. PPARy activation by rosiglitazone (RSG) was shown to improve the age-dependent decrease of LTP and neurocognitive deficits depending on aging in older animals [40]. Also, activation PPARY could modulate the activation of microglia and suppress NF-KB-mediated pro-inflammatory gene transcription, which inhibited inflammatory response and was beneficial for AD [41]. In the present study, we used RSG as a positive drug, and demonstrated RSG significantly reduced the LPS-induced inflammatory response in BV-2 cells, consistent with the effect of salidroside and AMPK agonist metformin in LPS-induced BV-2 cells. And here salidroside inhibited the AMPK/ PPARy signaling pathway and thereby exhibited antineuroinflammatory activity in cultured microglial cells in this study.

In addition to the suppression of neuroinflammation, our in vivo and in vitro results suggested that the therapeutic effects of salidroside may also be mediated via the switching of microglial phenotype. There are two different phenotypes of activated microglia: classically activated M1 phenotypes and alternatively activated $\mathrm{M} 2$ phenotypes. $\mathrm{M} 1$ microglia secretes pro-inflammatory cytokines, such as interleukins-1 $\beta$ (IL-1 $\beta$ ), IL-6, and tumor necrosis factor-a (TNF-a), which promote inflammation; while M2 microglia secrete anti-inflammatory cytokines, such as IL-10, IL-4, and transforming growth factor- $\beta$ (TGF$\beta$ ), which inhibit inflammation and promote tissue repair [42]. It has been proven that promoting microglia to the M2 phenotype significantly improve postoperative cognition $[43,44]$. Thence, promoting the polarization of microglia to $\mathrm{M} 2$ type is an excellent strategy to improve PND. Changes in microglial phenotype were closely associated with the neuroprotective effect of salidroside. Microglial activation and the expression of inflammatory molecules are directly involved in neurodegenerative pathology and in the development of neuroinflammation. We observed an ameliorated inflammatory milieu by salidroside in microglial culture, histological assays of brain tissue from mice also showed that salidroside significantly attenuated microglial inflammatory activation in the hippocampus. Interestingly, we further observed that salidroside switched microglial phenotype from the inflammatory phenotype to the anti-inflammatory phenotype in vivo and in vitro. Furthermore, our results suggest that the salidrosideinduced switching of microglial phenotype towards the anti-inflammatory phenotype was mediated by AMPK/ PPARy signaling pathway in microglia.

Although we demonstrated that salidroside ameliorated behavioral impairments and neuropathological changes in a mouse model of PND by activating AMPK/ PPARY signaling, the specific binding site of salidroside remains unknown. In addition, we cannot rule out the possible effect of salidroside on other cells, but we have confirmed through in vitro experiments and immunofluorescence double-labeling tests that the effect of salidroside on PND is mainly through the regulation of microglia polarization.

\section{Conclusions}


In summary, we demonstrated that salidroside prevented surgery-induced neuroinflammation and cognitive deficits in the mouse model of PND. Notably, salidroside induced the polarization of microglia to an anti-inflammatory phenotype through regulating the AMPK/PPARY signaling pathway. These results suggest that salidroside may be a novel therapeutic approach for PND.

\section{Abbreviations}

AMPK, Adenosine 5'-monophosphate-activated protein kinase; PND, Perioperative neurocognitive disorders; MAPK, mitogen-activated protein kinases; NF-кB, Nuclear factor kappa B; LPS, Lipopolysaccharide; IL-1 $\beta$, Interleukin-1 $\beta$; ERK, Extracellular-signal regulated kinase; PI3K, Phosphoinositide 3-kinase; HIFa, hypoxia-inducible factor $\alpha$; AD, Alzheimer's disease; MMPs, Matrix metalloproteinases; NRF2, Nuclear factor erythroid 2-related factor 2; HO-1, Heme oxygenase-1; PPARY, Peroxisome proliferators-activated receptor $\mathrm{y}$; MWM, Morris water maze; TNF-a, Tumor necrosis factor-a; IFN- $\gamma$, Interferon- $\gamma$; CCL5, CC chemokine ligand 5; CXCL1, Chemokine (C-X-C motif) ligand 1 ; TGF- $\beta$, Transforming growth factor- $\beta$; Arg1, Arginase1; YM1, Chitinase 3-like 3.

\section{Declarations}

\section{Ethics approval and consent to participate}

All experimental protocols and procedures were approved and licensed by Nanjing Medical University Animal Care and Use Committee in accordance with the National Institutes of Health guide for the care and use of Laboratory animals.

\section{Consent for publication}

Not applicable.

\section{Availability of data and material}

The datasets generated and/or analyzed during the current study are available from the corresponding author on reasonable request.

\section{Competing interests}

The authors declare that they have no competing interests.

\section{Funding}


This work was supported by the National Natural Science Foundation of China (Grant No. 81771171), Nanjing Medical Science and Technology Development Foundation (Grant No. YKK19076, YKK19081), Science and Technology Development Foundation of Nanjing Medical University (Grant No. NMUB2019002, NMUB2019172), and a grant (Grant No. 2016YFA0502201) from the National Key Research Program of China.

\section{Authors' contributions}

$Z-Y Z$ designed the experiments, obtained resources, and acquired funding. Z-YZ conducted the experiments with assistance from C-LP and G-LD. C-LP, G-LD, H-WZ, C-YZ, Q-HM, LX, NX, XL, ZZ, YZ, Q-LT, $X-L W$ and $Z-Y Z$ collected the data and contributed to the statistical analysis. C-LP, G-LD, and Z-YZ analyzed the data and wrote the manuscript. All authors read and approved the final manuscript.

\section{Acknowledgements}

Not applicable.

\section{References}

1. Evered L, Silbert B, Knopman DS, Scott DA, DeKosky ST, Rasmussen LS, Oh ES, Crosby G, Berger M, Eckenhoff RG. Nomenclature Consensus Working G: Recommendations for the nomenclature of cognitive change associated with anaesthesia and surgery-2018. Br J Anaesth. 2018;121:1005-12.

2. Monk TG, Weldon BC, Garvan CW, Dede DE, van der Aa MT, Heilman KM, Gravenstein JS. Predictors of cognitive dysfunction after major noncardiac surgery. Anesthesiology. 2008;108:18-30.

3. Zywiel MG, Hurley RT, Perruccio AV, Hancock-Howard RL, Coyte PC, Rampersaud YR. Health economic implications of perioperative delirium in older patients after surgery for a fragility hip fracture. J Bone Joint Surg Am. 2015;97:829-36.

4. Steinmetz J, Christensen KB, Lund T, Lohse N, Rasmussen LS, Group I. Long-term consequences of postoperative cognitive dysfunction. Anesthesiology. 2009;110:548-55.

5. Subramaniyan S, Terrando N. Neuroinflammation and Perioperative Neurocognitive Disorders. Anesth Analg. 2019;128:781-8.

6. Feng X, Valdearcos M, Uchida Y, Lutrin D, Maze M, Koliwad SK. Microglia mediate postoperative hippocampal inflammation and cognitive decline in mice. JCI Insight. 2017;2:e91229.

7. Wang HL, Liu H, Xue ZG, Liao QW, Fang H. Minocycline attenuates post-operative cognitive impairment in aged mice by inhibiting microglia activation. J Cell Mol Med. 2016;20:1632-9.

8. Forsberg A, Cervenka S, Jonsson Fagerlund M, Rasmussen LS, Zetterberg $H$, Erlandsson Harris $H$, Stridh P, Christensson E, Granstrom A, Schening A, et al. The immune response of the human brain to abdominal surgery. Ann Neurol. 2017;81:572-82. 
9. Liu Y, Yin Y. Emerging Roles of Immune Cells in Postoperative Cognitive Dysfunction. Mediators Inflamm. 2018;2018:6215350.

10. Tao H, Wu X, Cao J, Peng Y, Wang A, Pei J, Xiao J, Wang S, Wang Y. Rhodiola species: A comprehensive review of traditional use, phytochemistry, pharmacology, toxicity, and clinical study. Med Res Rev. 2019;39:1779-850.

11. Panossian A, Wikman G, Sarris J. Rosenroot (Rhodiola rosea): traditional use, chemical composition, pharmacology and clinical efficacy. Phytomedicine. 2010;17:481-93.

12. Nabavi SF, Braidy N, Orhan IE, Badiee A, Daglia M, Nabavi SM. Rhodiola rosea L. and Alzheimer's Disease: From Farm to Pharmacy. Phytother Res. 2016;30:532-9.

13. Borgonetti V, Governa P, Biagi M, Dalia P, Corsi L. Rhodiola rosea L. modulates inflammatory processes in a CRH-activated BV2 cell model. Phytomedicine. 2020;68:153143.

14. Zhong Z, Han J, Zhang J, Xiao Q, Hu J, Chen L. Pharmacological activities, mechanisms of action, and safety of salidroside in the central nervous system. Drug Des Devel Ther. 2018;12:1479-89.

15. Guan S, Feng H, Song B, Guo W, Xiong Y, Huang G, Zhong W, Huo M, Chen N, Lu J, Deng X. Salidroside attenuates LPS-induced pro-inflammatory cytokine responses and improves survival in murine endotoxemia. Int Immunopharmacol. 2011;11:2194-9.

16. Ding XJ, Zhang ZY, Jin J, Han JX, Wang Y, Yang K, Yang YY, Wang HQ, Dai XT, Yao C, et al. Salidroside can target both P4HB-mediated inflammation and melanogenesis of the skin. Theranostics. 2020;10:11110-26.

17. Zhang $Y$, Zhao Q. Salidroside attenuates interleukin-1beta-induced inflammation in human osteoarthritis chondrocytes. J Cell Biochem 2018.

18. Su Y, Zong S, Wei C, Song F, Feng H, Qin A, Lian Z, Fu F, Shao S, Fang F, et al. Salidroside promotes rat spinal cord injury recovery by inhibiting inflammatory cytokine expression and NF-kappaB and MAPK signaling pathways. J Cell Physiol. 2019;234:14259-69.

19. Wang C, Wang Q, Lou Y, Xu J, Feng Z, Chen Y, Tang Q, Zheng G, Zhang Z, Wu Y, et al. Salidroside attenuates neuroinflammation and improves functional recovery after spinal cord injury through microglia polarization regulation. J Cell Mol Med. 2018;22:1148-66.

20. Wang H, Li Q, Sun S, Chen S. Neuroprotective Effects of Salidroside in a Mouse Model of Alzheimer's Disease. Cell Mol Neurobiol. 2020;40:1133-42.

21. Zhang B, Wang Y, Li H, Xiong R, Zhao Z, Chu X, Li Q, Sun S, Chen S. Neuroprotective effects of salidroside through PI3K/Akt pathway activation in Alzheimer's disease models. Drug Des Devel Ther. 2016;10:1335-43.

22. Fan F, Yang L, Li R, Zou X, Li N, Meng X, Zhang Y, Wang X. Salidroside as a potential neuroprotective agent for ischemic stroke: a review of sources, pharmacokinetics, mechanism and safety. Biomed Pharmacother. 2020;129:110458.

23. Xu N, Huang F, Jian C, Qin L, Lu F, Wang Y, Zhang Z, Zhang Q. Neuroprotective effect of salidroside against central nervous system inflammation-induced cognitive deficits: A pivotal role of sirtuin 1dependent Nrf-2/HO-1/NF-kappaB pathway. Phytother Res. 2019;33:1438-47. 
24. Woolf AD, Akesson K. Preventing fractures in elderly people. BMJ. 2003;327:89-95.

25. Leme LE, Sitta Mdo C, Toledo M, da Silva Henriques S. Orthopedic Surgery among the Elderly: Clinical Characteristics. Rev Bras Ortop. 2011;46:238-46.

26. Xiong C, Zhang Z, Baht GS, Terrando N. A Mouse Model of Orthopedic Surgery to Study Postoperative Cognitive Dysfunction and Tissue Regeneration. J Vis Exp 2018.

27. Pan C, Si Y, Meng Q, Jing L, Chen L, Zhang Y, Bao H. Suppression of the RAC1/MLK3/p38 Signaling Pathway by beta-Elemene Alleviates Sepsis-Associated Encephalopathy in Mice. Front Neurosci. 2019;13:358.

28. Wang Y, Huang Y, Xu Y, Ruan W, Wang H, Zhang Y, Saavedra JM, Zhang L, Huang Z, Pang T. A Dual AMPK/Nrf2 Activator Reduces Brain Inflammation After Stroke by Enhancing Microglia M2 Polarization. Antioxid Redox Signal. 2018;28:141-63.

29. Villapol S. Roles of Peroxisome Proliferator-Activated Receptor Gamma on Brain and Peripheral Inflammation. Cell Mol Neurobiol. 2018;38:121-32.

30. Zheng T, Bian F, Chen L, Wang Q, Jin S. Beneficial Effects of Rhodiola and Salidroside in Diabetes: Potential Role of AMP-Activated Protein Kinase. Mol Diagn Ther. 2019;23:489-98.

31. Barhwal K, Das SK, Kumar A, Hota SK, Srivastava RB. Insulin receptor A and Sirtuin 1 synergistically improve learning and spatial memory following chronic salidroside treatment during hypoxia. $J$ Neurochem. 2015;135:332-46.

32. Zhang J, Zhen YF, Pu Bu Ci R, Song LG, Kong WN, Shao TM, Li X, Chai XQ. Salidroside attenuates beta amyloid-induced cognitive deficits via modulating oxidative stress and inflammatory mediators in rat hippocampus. Behav Brain Res. 2013;244:70-81.

33. Zhu L, Chen T, Chang X, Zhou R, Luo F, Liu J, Zhang K, Wang Y, Yang Y, Long H, et al. Salidroside ameliorates arthritis-induced brain cognition deficits by regulating Rho/ROCK/NF-kappaB pathway. Neuropharmacology. 2016;103:134-42.

34. Liang L, Ma Z, Dong M, Ma J, Jiang A, Sun X. Protective effects of salidroside against isofluraneinduced cognitive impairment in rats. Hum Exp Toxicol. 2017;36:1295-302.

35. Wang X, Zimmermann HR, Ma T. Therapeutic Potential of AMP-Activated Protein Kinase in Alzheimer's Disease. J Alzheimers Dis. 2019;68:33-8.

36. Salminen A, Kaarniranta K, Haapasalo A, Soininen H, Hiltunen M. AMP-activated protein kinase: a potential player in Alzheimer's disease. J Neurochem. 2011;118:460-74.

37. Du LL, Chai DM, Zhao LN, Li XH, Zhang FC, Zhang HB, Liu LB, Wu K, Liu R, Wang JZ, Zhou XW. AMPK activation ameliorates Alzheimer's disease-like pathology and spatial memory impairment in a streptozotocin-induced Alzheimer's disease model in rats. J Alzheimers Dis. 2015;43:775-84.

38. Liu B, Huang B, Hu G, He D, Li Y, Ran X, Du J, Fu S, Liu D. Isovitexin-Mediated Regulation of Microglial Polarization in Lipopolysaccharide-Induced Neuroinflammation via Activation of the CaMKKbeta/AMPK-PGC-1alpha Signaling Axis. Front Immunol. 2019;10:2650. 
39. d'Angelo M, Castelli V, Catanesi M, Antonosante A, Dominguez-Benot R, Ippoliti R, Benedetti E, Cimini A. PPARgamma and Cognitive Performance. Int J Mol Sci 2019, 20.

40. Xu L, Ma X, Verma N, Perie L, Pendse J, Shamloo S, Marie Josephson A, Wang D, Qiu J, Guo M, et al. PPARgamma agonists delay age-associated metabolic disease and extend longevity. Aging Cell. 2020;19:e13267.

41. Khan MA, Alam Q, Haque A, Ashafaq M, Khan MJ, Ashraf GM, Ahmad M. Current Progress on Peroxisome Proliferator-activated Receptor Gamma Agonist as an Emerging Therapeutic Approach for the Treatment of Alzheimer's Disease: An Update. Curr Neuropharmacol. 2019;17:232-46.

42. Colonna M, Butovsky O. Microglia Function in the Central Nervous System During Health and Neurodegeneration. Annu Rev Immunol. 2017;35:441-68.

43. Lee JH, Kam EH, Kim SY, Cheon SY, Kim EJ, Chung S, Jeong JH, Koo BN. Erythropoietin Attenuates Postoperative Cognitive Dysfunction by Shifting Macrophage Activation toward the M2 Phenotype. Front Pharmacol. 2017;8:839.

44. Pena-Altamira E, Petralla S, Massenzio F, Virgili M, Bolognesi ML, Monti B. Nutritional and Pharmacological Strategies to Regulate Microglial Polarization in Cognitive Aging and Alzheimer's Disease. Front Aging Neurosci. 2017;9:175.

\section{Table}


Table. 1 The primers used for RT-qPCR in the present study.

\begin{tabular}{|c|c|c|}
\hline Gene & Forward & Reverse \\
\hline Mouse IL-1 $\beta$ Mouse IL-1 $\beta$ & TGATGTGCTCACTGCCTGGTTTC & GTTGATGTGCTGCTGCGAGATTTG \\
\hline Mouse IL-4 & TGAATGACTGGAGGAGCTGAGACC & CGGAGTGGCGACATCGTACATAAC \\
\hline Mouse IL-6 & TACCACTTCACAAGTCGGAGGC & CTGCAAGTGCATCATCGTTGTTC \\
\hline Mouse IL-10 & TTCTTTCAAACAAAGGACCAGC & GCAACCCAAGTAACCCTTAAAG \\
\hline Mouse IL-12 & GACCTGTTTACCACTGGAACTA & GATCTGCTGATGGTTGTGATTC \\
\hline Mouse TNF-a & ATGTCTCAGСCTCTTCTCATTC & GCTTGTCACTCGAATTTTGAGA \\
\hline Mouse IFN- $\gamma$ & CTTGAAAGACAATCAGGCCATC & CTTGGCAATACTCATGAATGCA \\
\hline Mouse CCL5 & GTATTTCTACACCAGCAGCAAG & TCTTGAACCCACTTCTTCTCTG \\
\hline Mouse CXCL1 & ATGGCTGGGATTCACCTCAAGAAC & AGTGTGGCTATGACTTCGGTTTGG \\
\hline Mouse CXCL10 & CAACTGCATCCATATCGATGAC & GATTCCGGATTCAGACATCTCT \\
\hline Mouse CD16 & TTCAGCCACACAGCCTTCCTTTG & ССТССТTССTCAGССTССTAAGTC \\
\hline Mouse TGF- $\beta$ & CCAGATCCTGTCCAAACTAAGG & CTCTTTAGCATAGTAGTCCGCT \\
\hline Mouse Arg1 & CATATCTGCCAAAGACATCGTG & GACATCAAAGCTCAGGTGAATC \\
\hline Mouse YM1 & CAGTGTTCTGGTGAAGGAAATG & ACCCAGACTTGATTACGTCAAT \\
\hline Mouse GAPDH & CATCACTGCCACCCAGAAGACTG & ATGCCAGTGAGCTTCCCGTTCAG \\
\hline
\end{tabular}

\section{Figures}



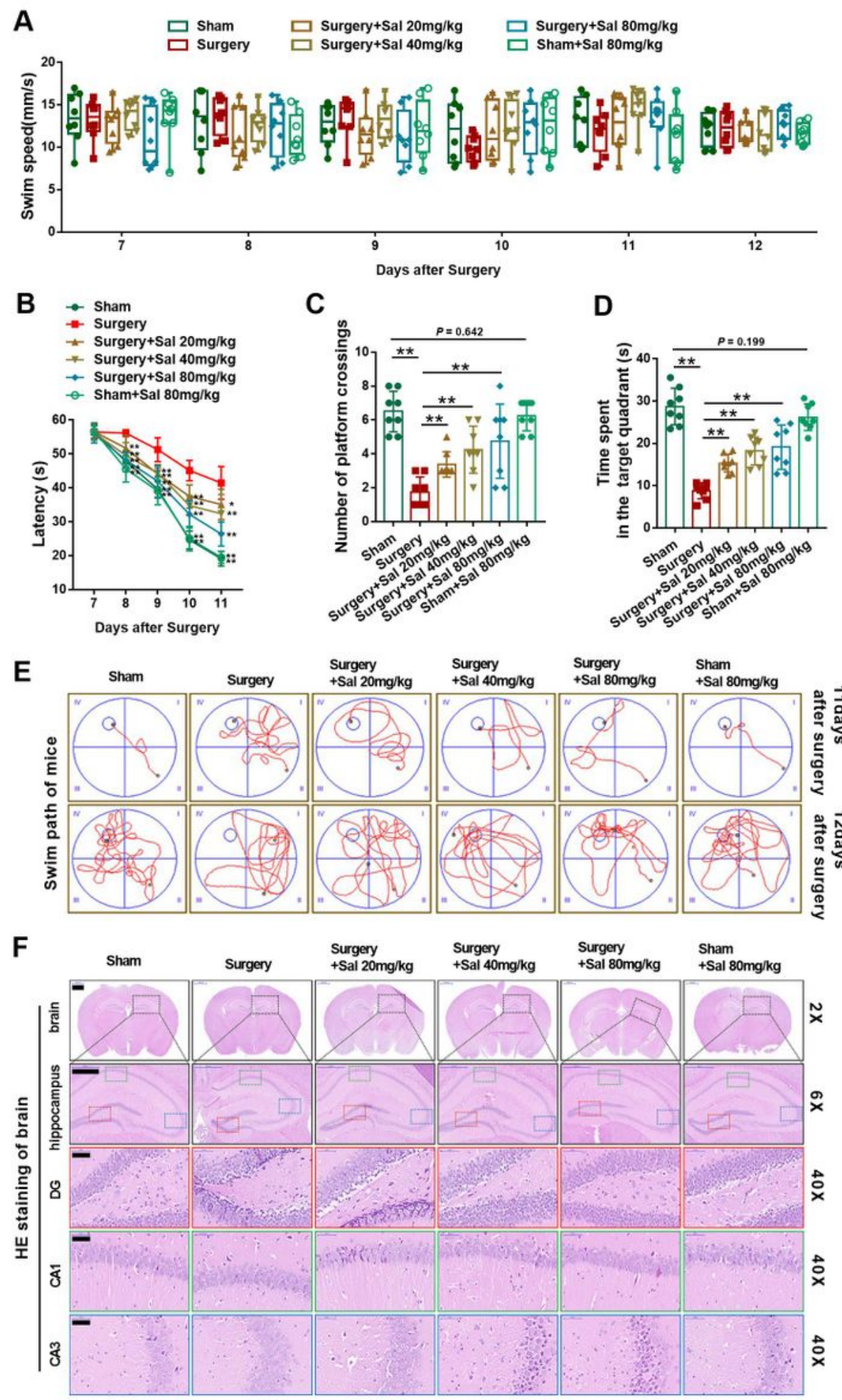

\section{Figure 1}

Salidroside improves functional recovery after orthopedic surgery. Sham operation or orthopedic surgery were carried out in 15 months old male C57BL/ 6 mice. 20,40 or $80 \mathrm{mg} / \mathrm{kg}$ Salidroside was intraperitoneally injected to surgery mice. $80 \mathrm{mg} / \mathrm{kg}$ Salidroside was intraperitoneally injected to sham mice. MWM test was used to detect the swim speed (A), latency (B), platform crossing times (C), and time spent in target quadrant (D) at the indicated time. Representative trace graphs of mice in the MWM test 
showed the swim path at the indicated time (E). H\&E staining was used to observe the pathological changes in brain in mice (F). Scale bars were $1000 \mu \mathrm{m}(2 \times), 500 \mu \mathrm{m}(6 \times)$ and $50 \mu \mathrm{m}(40 x)$ in representative graphs. Data are presented as the mean \pm standard deviation, $n=8$. Compared with model group, $* P<0.05$, and ${ }^{*} \mathrm{P}<0.01$.

A
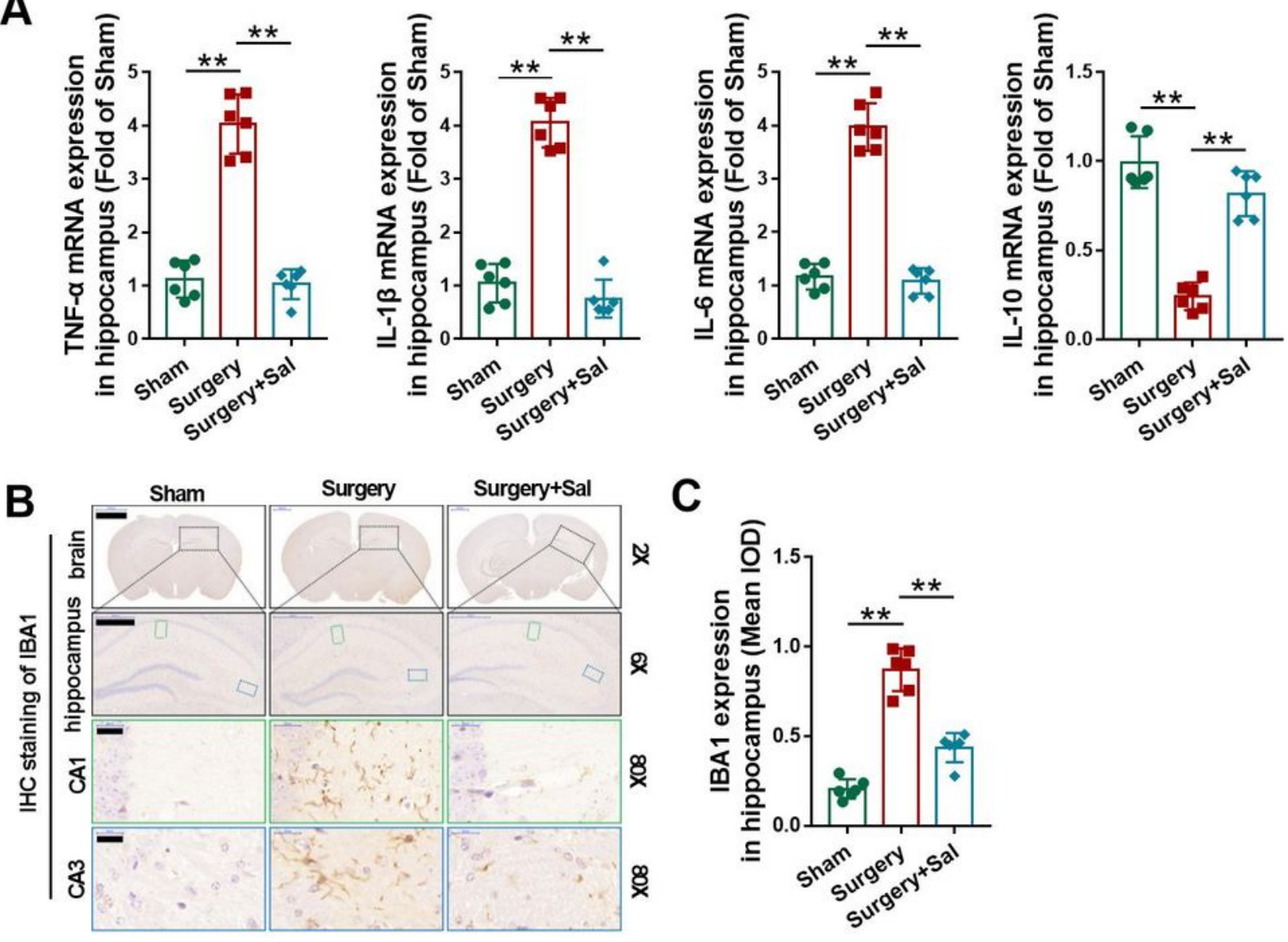

C
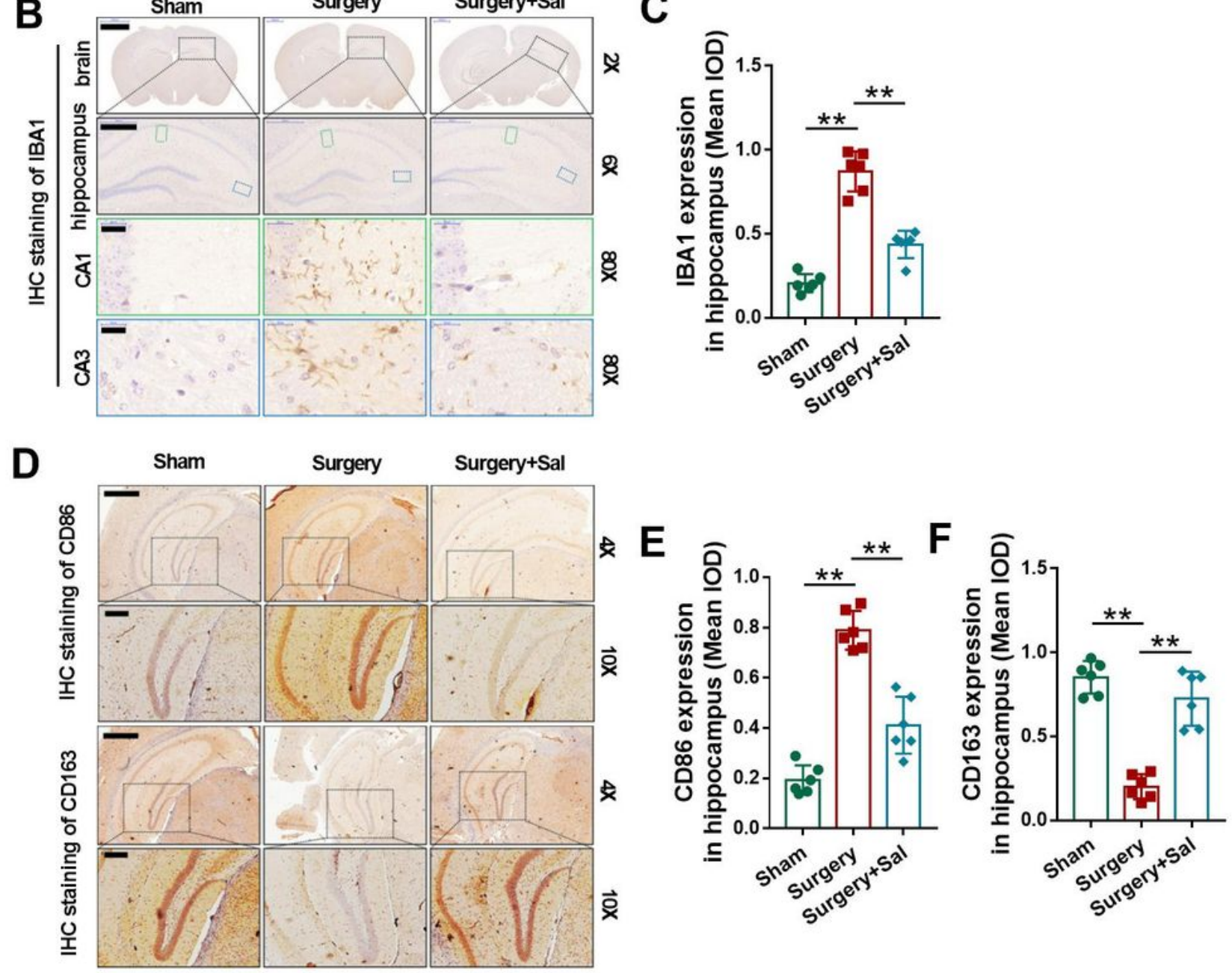

Figure 2 
Salidroside inhibits orthopedic surgery-induced neuroinflammation in mice. Sham operation or orthopedic surgery were carried out in 15 months old male C57BL/6 mice. Salidroside $(80 \mathrm{mg} / \mathrm{kg})$ was intraperitoneally injected to surgery mice. RT-qPCR analysis was used to detect the mRNA expression of inflammatory factors TNF-a, IL-1 $\beta$, IL-6 and IL-10 (A) in hippocampus in mice. Representative immunohistochemical staining graphs (B) and optical density analysis (C) of IBA1 in hippocampus in mice showed the activation of microglia. Representative immunohistochemical staining graphs (D) and optical density analysis of CD86 (E) and CD163 (F) in hippocampus in mice showed the M1 or M2 status of microglia. Scale bars were $1000 \mu \mathrm{m}(2 \times), 800 \mu \mathrm{m}(4 \times), 500 \mu \mathrm{m}(6 \times), 250 \mu \mathrm{m}(10 x)$, and $25 \mu \mathrm{m}(80 x)$ in representative graphs. Data are presented as the mean \pm standard deviation, $n=6$. ${ }^{*} P<0.05$, and $* \star P<$ 0.01 between the indicated groups. 

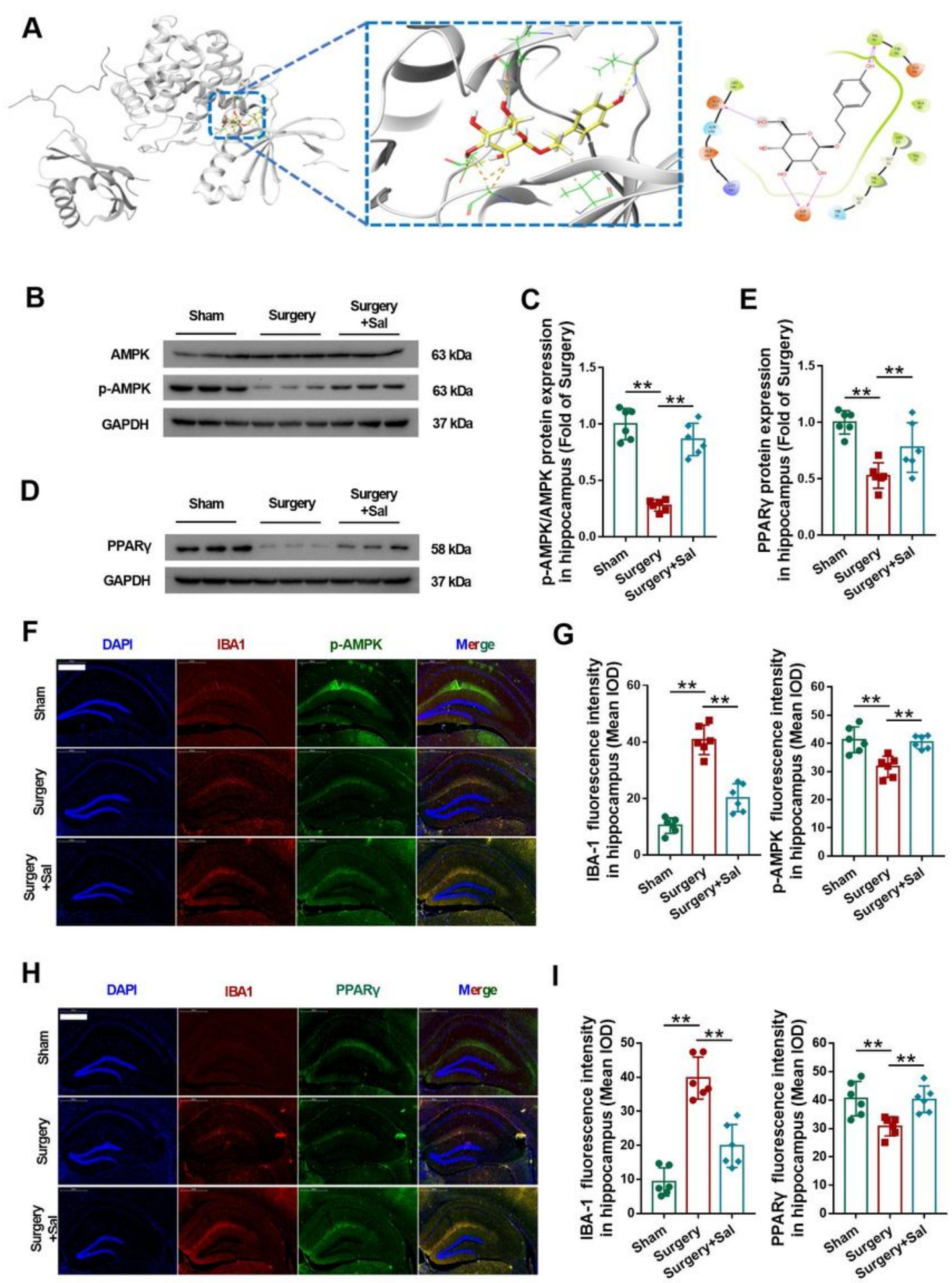

\section{Figure 3}

AMPK and PPARy participates in the neuroprotective effect of salidroside. Molecular docking profiled the interaction diagram between Salidrosid and AMPK(A). Sham operation or orthopedic surgery were carried out in 15 months old male C57BL/6 mice. $80 \mathrm{mg} / \mathrm{kg}$ Salidroside was intraperitoneally injected to surgery mice. Western blot (B) and relative quantitative analysis (C) were used to reflect the protein expression of AMPK and p-AMPK in hippocampus in mice. Western blots (D) and relative quantitative analysis (E) for 
PPARy protein expression in each group of mice were also carried out. Representative immunofluorescence staining graphs (F) and fluorescence density analysis (G) of p-AMPK (red) and IBA1 (green) in hippocampus in mice showed the activation of microglia and AMPK signal. Representative immunofluorescence staining graphs $(\mathrm{H})$ and fluorescence density analysis $(\mathrm{I})$ of PPARY (red) and IBA1 (green) in hippocampus in mice showed the activation of microglia and PPARY signal. Scale bars were $500 \mu \mathrm{m}(6 \times)$ in representative graphs. Data are presented as the mean \pm standard deviation, $n=6 . * P<$ 0.05 , and $* * P<0.01$ between the indicated groups.

A

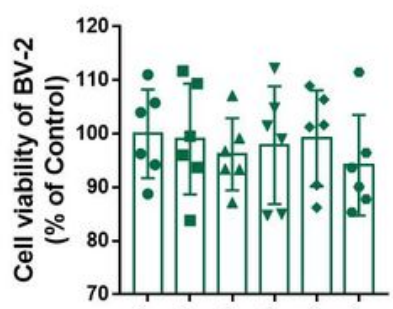

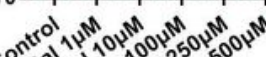

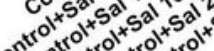

D

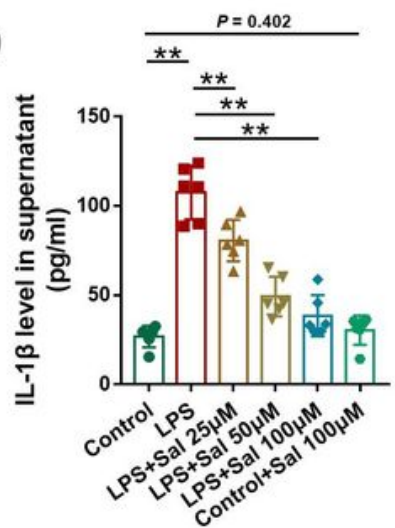

G
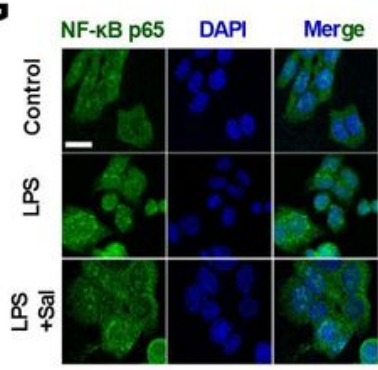

H

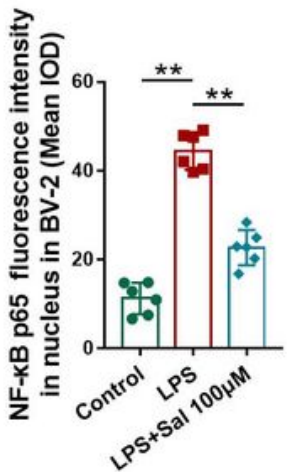

B

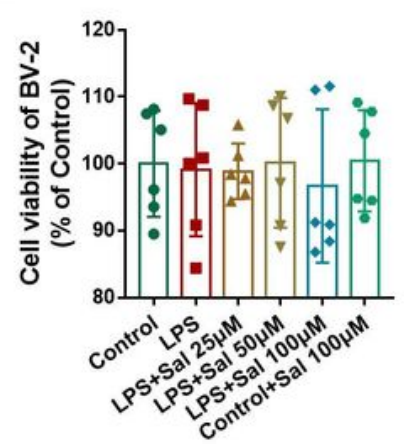

E
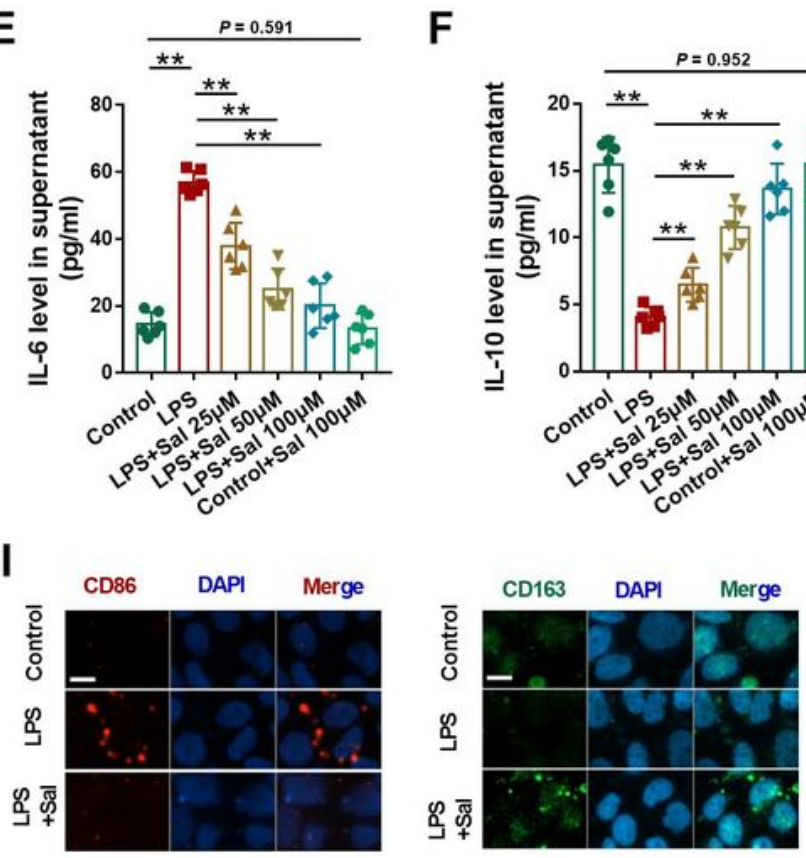

F
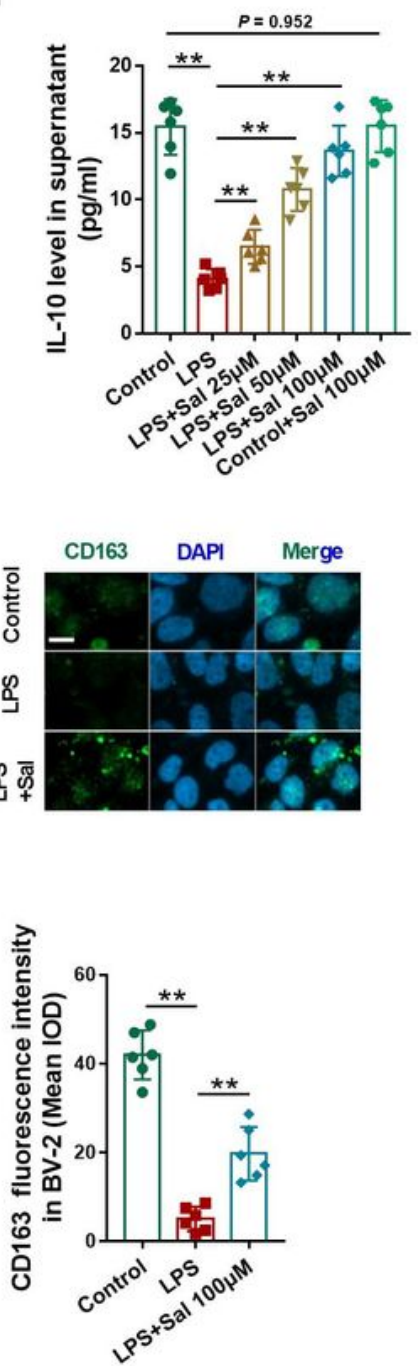


\section{Figure 4}

Salidroside inhibits LPS-induced inflammatory response in BV-2 cells and promotes its polarization to M2 phenotype. CCK8 measurement was used to detect cell vitality in normal BV-2 cells treated with different concentrations of Salidroside $(0 \mu \mathrm{M}, 1 \mu \mathrm{M}, 10 \mu \mathrm{M}, 100 \mu \mathrm{M}, 250 \mu \mathrm{M}$, and $500 \mu \mathrm{M})$ for $24 \mathrm{~h}$ (A). Cell vitality was also detected in normal BV-2 cells pretreated with or without $100 \mu \mathrm{M}$ Salidroside and in LPS (1 $\mu \mathrm{g} / \mathrm{ml})$ induced BV-2 cells pretreated with or without $(25,50$, and $100 \mu \mathrm{M})$ salidroside for $12 \mathrm{~h}(\mathrm{~B})$. TNF-a (C), IL-1 $\beta(D)$, IL-6 (E), and IL-10 (F) release was detected by ELISA in culture media from normal BV-2 cells pretreated with or without $100 \mu \mathrm{M}$ Salidroside, and in culture media from LPS $(1 \mu \mathrm{g} / \mathrm{ml})$ induced BV2 cells pretreated with or without $(25,50$, and $100 \mu \mathrm{M})$ salidroside for $12 \mathrm{~h}$. Representative immunofluorescent staining graphs $(\mathrm{G})$ and optical density analysis $(\mathrm{H})$ of NF-kB p65 in normal BV-2 cells and in LPS $(1 \mu \mathrm{g} / \mathrm{ml})$ induced BV-2 cells pretreated with or without $100 \mu \mathrm{M}$ salidroside for $12 \mathrm{~h}$ showed the nuclear translocation of NF-kB p65. Representative immunofluorescent staining graphs (I) and optical density analysis $(J)$ of CD86 and CD163 showed the M1 or M2 status in normal BV-2 cells and in LPS $(1 \mu \mathrm{g} / \mathrm{ml})$ induced BV-2 cells pretreated with or without $100 \mu \mathrm{M}$ salidroside for $12 \mathrm{~h}$. Scale bars were $25 \mu \mathrm{m}(40 \times)$ in representative graphs. Data are presented as the mean \pm standard deviation, $n=$ 6. ${ }^{*} \mathrm{P}<0.05$, and ${ }^{\star \star} \mathrm{P}<0.01$ between the indicated groups. 
A

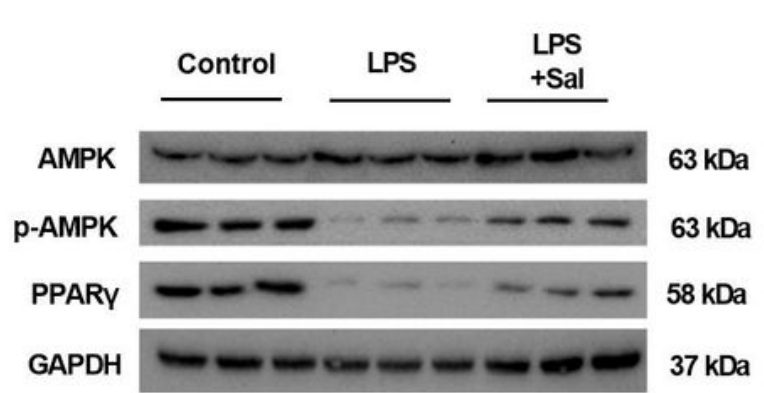

B
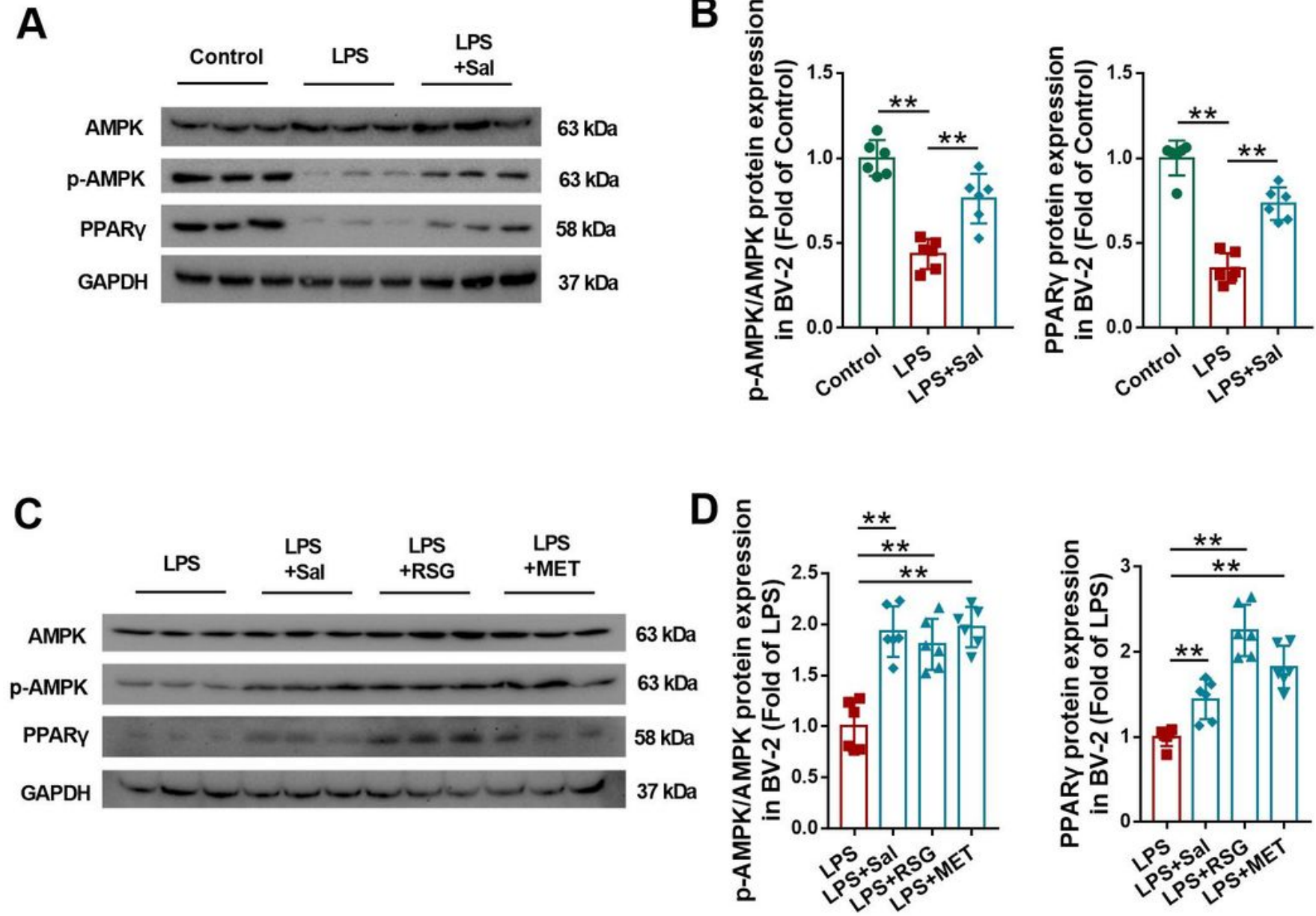

$\mathbf{E}$

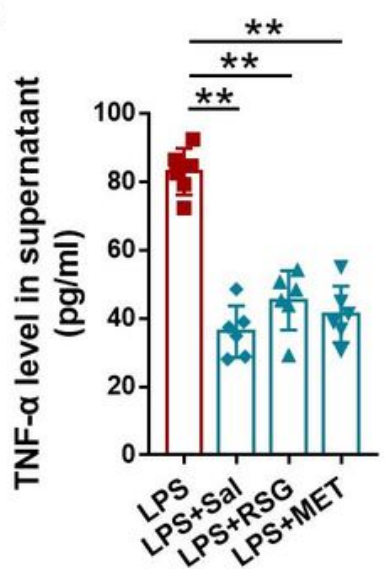

$\mathbf{F}$

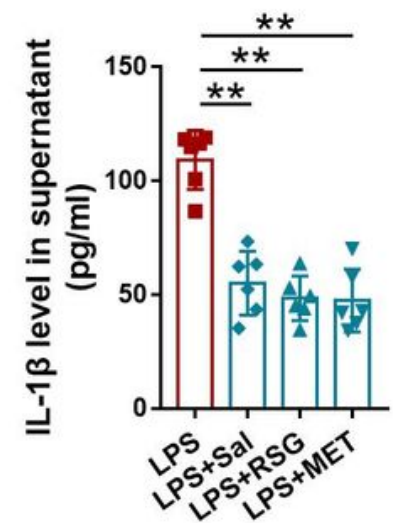

G

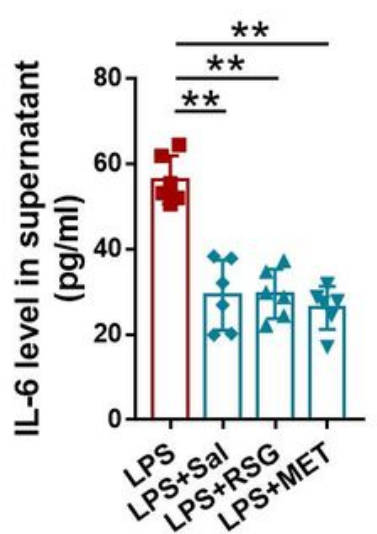

H

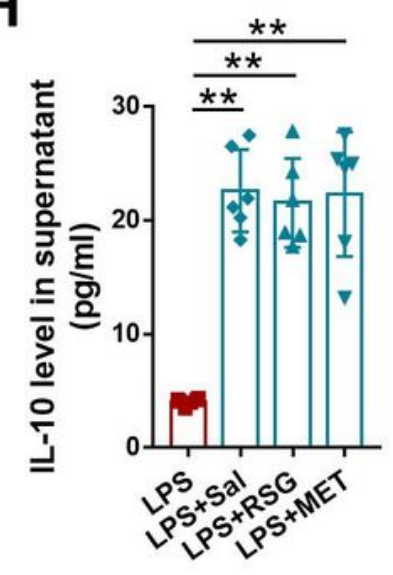

Figure 5

The AMPK/PPARy pathway is involved in anti-inflammatory effect of Salidroside in BV-2 cells. Western blot $(A)$ and relative quantitative analysis $(B)$ were used to reflect the protein expression of AMPK, $p$ AMPK, PPARy in normal BV-2 cells and in LPS $(1 \mu \mathrm{g} / \mathrm{ml})$ induced BV-2 cells pretreated with or without 100 $\mu \mathrm{M}$ salidroside for $12 \mathrm{~h}$. Western blot (C) and relative quantitative analysis (D) were used to reflect the protein expression of AMPK, p-AMPK, PPARy in LPS $(1 \mu \mathrm{g} / \mathrm{ml})$ induced BV-2 cells pretreated with or 
without $100 \mu \mathrm{M}$ salidroside, $10 \mu \mathrm{M}$ rosiglitazone (RSG), or $10 \mu \mathrm{M}$ metformin (MET) for $12 \mathrm{~h}$. TNF-a (E), IL$1 \beta(F), I L-6(G)$, and IL-10 $(H)$ release was detected by ELISA in culture media from LPS $(1 \mu \mathrm{g} / \mathrm{ml})$ induced BV-2 cells pretreated with or without $100 \mu \mathrm{M}$ salidroside, $10 \mu \mathrm{M}$ rosiglitazone (RSG), or $10 \mu \mathrm{M}$ metformin (MET) for $12 \mathrm{~h}$. Data are presented as the mean \pm standard deviation, $n=6$. ${ }^{*}<0.05$, and ${ }^{* * P}<0.01$ between the indicated groups.
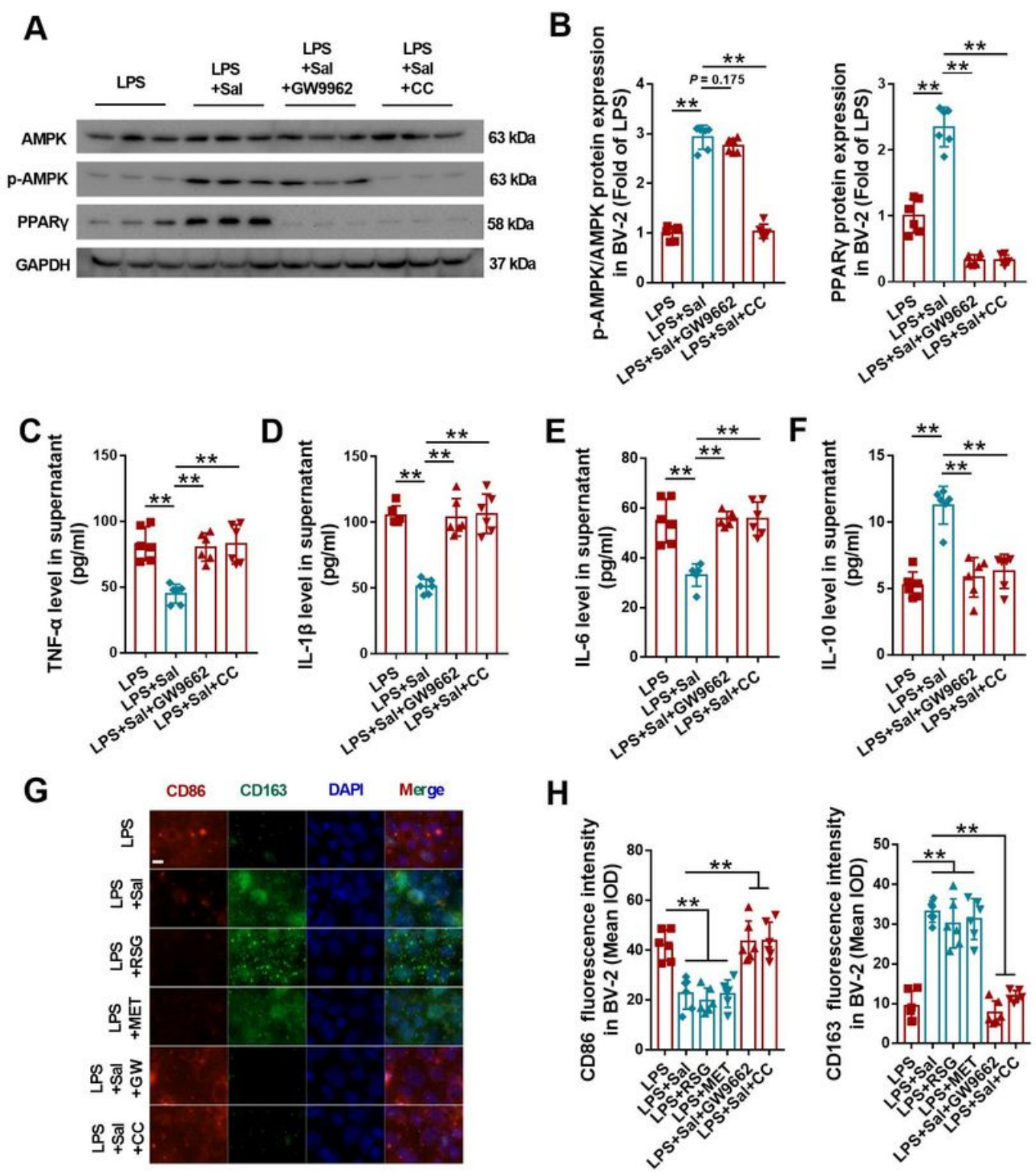

H

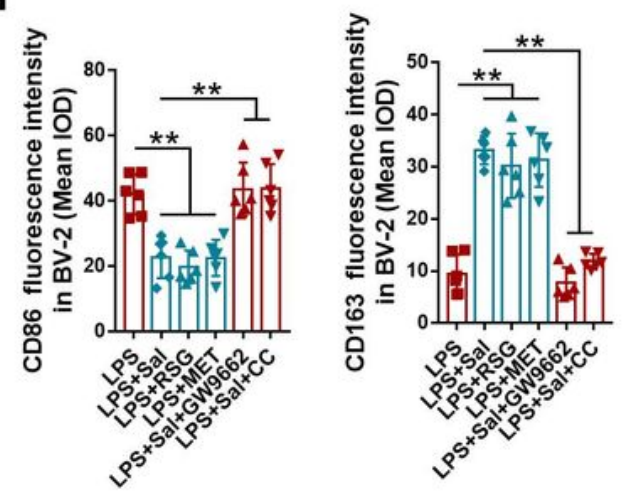

I

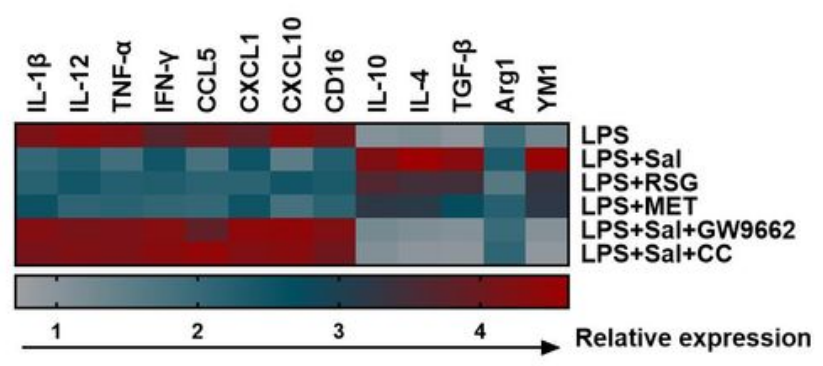

Figure 6 
The AMPK/PPARy pathway is involved in Salidroside-mediated microglia polarization in BV-2 cells. Western blot $(A)$ and relative quantitative analysis $(B)$ were used to reflect the protein expression of AMPK, p-AMPK, PPARy in LPS $(1 \mu \mathrm{g} / \mathrm{ml})$ induced BV-2 cells pretreated with or without $100 \mu \mathrm{M}$ salidroside combine with GW9662 or compand C (CC) incubation for $12 \mathrm{~h}$. TNF-a (C), IL-1ß (D), IL-6 (E), and IL-10 (F) release was detected by ELISA in culture media from LPS $(1 \mu \mathrm{g} / \mathrm{ml})$ induced BV-2 cells pretreated with or without $100 \mu \mathrm{M}$ salidroside combine with GW9662 or compand C (CC) incubation for $12 \mathrm{~h}$.

Representative immunofluorescent staining graphs $(G)$ and optical density analysis $(H)$ of CD86 and CD163 showed the M1 or M2 status in LPS $(1 \mu \mathrm{g} / \mathrm{ml})$ induced BV-2 cells pretreated with or without 100 $\mu \mathrm{M}$ salidroside, $10 \mu \mathrm{M}$ rosiglitazone (RSG), or $10 \mu \mathrm{M}$ metformin (MET) and in LPS (1 $\mu \mathrm{g} / \mathrm{ml})$ induced BV-2 cells pretreated with $100 \mu \mathrm{M}$ salidroside combine with $20 \mu \mathrm{M}$ GW9662 or $20 \mu \mathrm{M}$ compound C (CC) incubation for $12 \mathrm{~h}$. Scale bars were $25 \mu \mathrm{m}(40 \mathrm{x})$ in representative graphs. Heat map of RT-qPCR data showed the M1 and M2 marker genes (I) in LPS $(1 \mu \mathrm{g} / \mathrm{ml})$ induced BV-2 cells pretreated with or without $100 \mu \mathrm{M}$ salidroside, $10 \mu \mathrm{M}$ rosiglitazone (RSG), or $10 \mu \mathrm{M}$ metformin (MET) and in LPS $(1 \mu \mathrm{g} / \mathrm{ml}$ ) induced BV-2 cells pretreated with $100 \mu \mathrm{M}$ salidroside combine with $20 \mu \mathrm{M}$ GW9662 or $20 \mu \mathrm{M}$ compand C (CC) incubation for $12 \mathrm{~h}$. Red indicates high and blue indicates low levels of gene expression. Data are presented as the mean \pm standard deviation, $n=6$. ${ }^{*}<<0.05$, and $* * P<0.01$ between the indicated groups. 

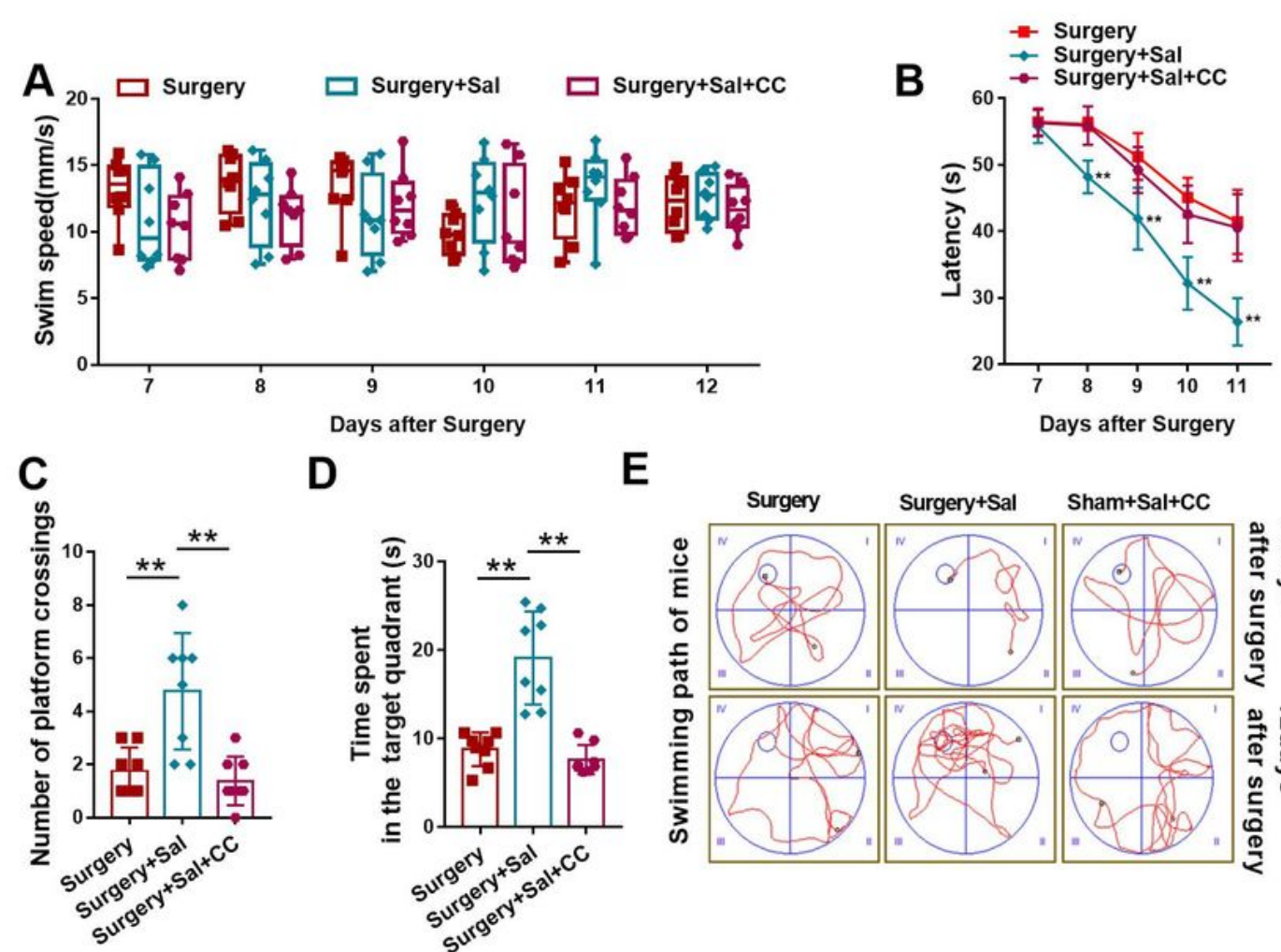

D
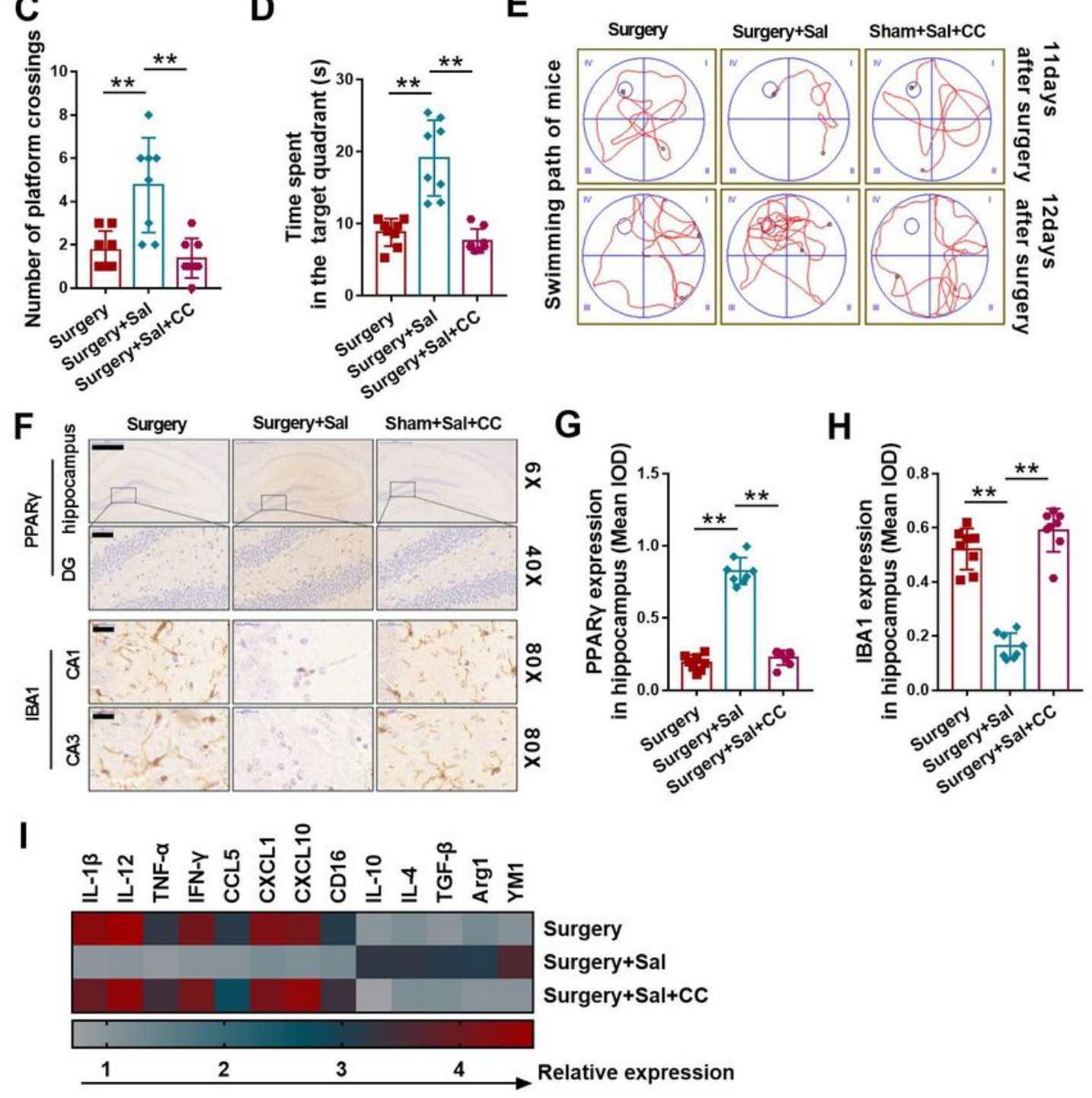

\section{Figure 7}

salidroside improved the cognitive impairment of fractured mice by AMPK/PPARY pathway. Orthopedic surgery was carried out in 15 months old male C57BL/ 6 mice. $80 \mathrm{mg} / \mathrm{kg}$ Salidroside with or without 5 $\mathrm{mg} / \mathrm{kg}$ compound $\mathrm{C}(\mathrm{CC})$ was intraperitoneally injected to surgery mice. MWM test was used to detect the swim speed (A), latency (B), platform crossing times (C), and time spent in target quadrant (D) at the indicated time. Representative trace graphs of mice in the MWM test showed the swim path at the 
indicated time $(E)$. Representative immunohistochemical staining graphs $(F)$ and optical density analysis of PPARY (G) and IBA1 $(\mathrm{H})$ in hippocampus in mice showed the PPARy expression and activation of microglia. Scale bars were $500 \mu \mathrm{m}(6 \times), 50 \mu \mathrm{m}(40 \times)$ and $25 \mu \mathrm{m}(80 \times)$ in representative graphs. Heat map of RT-qPCR data showed the M1 and M2 marker genes in hippocampus in mice (I). Red indicates high and blue indicates low levels of gene expression. Data are presented as the mean \pm standard deviation, $n$ $=8$. $* P<0.05$, and ${ }^{*} \mathrm{P}<0.01$ between the indicated groups.

\section{Supplementary Files}

This is a list of supplementary files associated with this preprint. Click to download.

- WB.pdf 